and visit count improved the selection of RA patients from a $67 \%$ to $90 \%$ accuracy. The combination of these variables provides a widely applicable algorithm, as they are broadly registered in Rheumatology clinics.

Subsequent replications are ongoing.

Disclosure of Interest: None declared

DOI: 10.1136/annrheumdis-2017-eular.6464

\section{THU0636 INFLUENZA AND MENINGOCOCCAL C VACCINATIONS IN A COHORT OF PATIENTS WITH AUTOIMMUNE RHEUMATIC DISEASES: ADHERENCE, SAFETY AND IMMUNOGENICITY}

R. Vagelli, C. Tani, L. Maggi, E. Elefante, A. Parma, E. Cioffi, S. Talarico, C. Baldini, C. Stagnaro, A. Delle Sedie, S. Vagnani, M. Mosca. Department of Clinical and Experimental Medicine, Rheumatology Unit, University of Pisa, Pisa, Italy

Background: The EULAR recommendations for vaccination in adult patients with autoimmune rheumatic diseases strongly recommend inactivated influenza vaccination.Insufficient data are available about safety and efficacy of meningococcal C vaccination. In 2015-2016, after an increased incidence of meningitidis C infections in our country,the health care system has promoted a free meningococcal vaccination campaign

Objectives: To evaluate the adherence to the EULAR recommendations for influenza vaccination and to the meningococcal $C$ vaccination campaign in a cohort of patients with autoimmune rheumatic diseases and to assess their safety. The efficacy in term of immune response to meningococcal $\mathrm{C}$ vaccination has been also evaluated

Methods: Consecutive in- and out-patients seen at our unit from February to December 2016 were enrolled in the study. Using a questionnaire created ad hoc the following data were collected:the percentage of patients who underwent influenza and/or meningococcal $\mathrm{C}$ vaccinations in the previous 12 months, the occurrence of adverse events and of disease flares after vaccinations,according with the report from the patients and with the rheumatologist clinical evaluation.Seroconvertion rates in patients and healthy controls were assessed using ELISA kits for human anti-meningococcal ACWY IgG antibodies.Antibody titres were expressed in $\mathrm{U} / \mathrm{ml}$ and according with kit reference value were classified in absent,low,medium and high titre

Results: 286 patients (91\% female) (143 SLE, 68 RA,60 Scleroderma, 11 Sjögren Syndrome, 3 Behcet disease and 1 Dermatomyositis) were included in the analysis. The mean age at evaluation was $52.9 \pm 16.1$ years, mean disease duration was $15.3 \pm 10$ years. The $53.1 \%$ of patients was taking steroids, at an average dose of $4.2 \mathrm{mg}$ of 6 -metilprednisolone/day,134/286 (46.9\%) patients were on immunosuppressive therapies, of which $49 / 134$ (36.6\%) on biologic agents. The $19.9 \%$ (57/286) of patients underwent influenza vaccinations and the $13.3 \%(38 / 286)$ meningococcal $\mathrm{C}$ vaccination, 8 patients underwent both vaccinations.No disease flares were observed after vaccination;seven patients reported non-specific adverse events after influenza (fever,discomfort,nausea,arthralgia) and 2 patients after meningococcal $C$ vaccination (fever,rash at the injection site,discomfort).Seroconversion after meningococcal vaccination was analysed in 27 patients and 9 healthy subjects,no statistically significant differences in terms of antibody response to meningococcal vaccination were observed between these two groups. Treatment (steroids and immunosuppressive drugs) did not influence antibody titres

Conclusions: These data highlight the poor adherence to international recommendations on influenza vaccination in patients with autoimmune rheumatic disease at our Unit. The adherence to the meningococcal $\mathrm{C}$ vaccination campaign conducted in our country in 2015-2016 was also low.Our data confirm the safety of these vaccination and show that the immune response elicited by meningococcal $C$ vaccination is comparable to healthy controls and is not influence by therapy References:

[1] van Assen S, Agmon-Levin N, Elkayam O, et al.EULAR recommendations for vaccination in adult patients with autoimmune inflammatory rheumatic diseases.Annals of the rheumatic diseases 2011; 70(3): 414-22.

Disclosure of Interest: None declared

DOI: 10.1136/annrheumdis-2017-eular.6123

\section{THU0637 PATIENT'S AND RHEUMATOLOGIST'S PERSPECTIVES ON THE FOLLOW-UP INTERVAL AS A TOOL FOR OPTIMIZED OUTPATIENT TREATMENT}

S. Hermann, S. Fügner, E. Wiebe, T. Alexander, G.R. Burmester, F. Buttgereit. Dept. of Rheumatology, Charite, Berlin, Germany

Background: Scientific progress and better disease awareness constantly lead to increasing patient numbers in rheumatology which requires optimization of patient care.

Objectives: The aim of this study was to evaluate and to optimize the procedures of patient care in an university-based outpatient rheumatology setting in Berlin, Germany.

Methods: One hundred patients with rheumatoid arthritis (80 women, 20 men, mean age 61.2 years, mean disease duration 12.9 years) were independently assessed both by a rheumatologist and via patient-reported self-assessment questionnaires. Current follow-up interval (usually 3 months), patient's perspective on follow-up intervals, signs of disease activity as well as individual patient concerns were recorded. Satisfaction with follow-up intervals was grouped into three categories: too early, just right/optimal, too late.

Results: Based on the physicians perspective, 46 patients presented at the optimal time point, 51 too early, and three too late. The patients reported the category "just right" in 82 cases, too early follow-up in 10 cases and too late in 8 cases. Of note, $51 \%$ (42 individuals) of all patients with self-reported satisfactory follow-up interval were judged to visit the out-patient department too early by the expert rheumatologist. When taking into account the follow-up interval and optimal satisfactory levels, $62 \%$ of patients were concluded to visit the department too early in those revisited after $3-4$ months $(n=65)$, and in $12 \%$ of those who were seen again after $5-6$ months $(n=17) .82 \%$ of patients in the latter group were judged to revisit just right by the physician.

Conclusions: There was a high proportion of overlap in the views on the satisfaction with follow-up intervals between physicians and patients. Especially in patients who were seen every $3 \mathbf{- 4}$ months, a high proportion was deemed to could have come later to the out-patient care unit from a purely medical point of view. Here we see a way to stretch the interval to 5-6 months without risking a long-term deterioration in patient care. However, this measure should be flanked by patient education and good collaboration with the general practitioners.

Acknowledgements: We thank AbbVie for financial support in the development of measures to optimize out-patient management in patients with rheumatoid arthritis. The sponsor did not influence the scientific results.

Disclosure of Interest: None declared

DOI: 10.1136/annrheumdis-2017-eular.4748

\section{THU0638 PREVALENCE AND DIRECT HEALTHCARE COSTS OF UPPER GASTROINTESTINAL (UGI) ADVERSE EVENTS IN ASIAN RHEUMATIC PATIENTS ON LONG-TERM NON-STEROIDAL ANTI-INFLAMMATORY DRUGS (NSAIDS)}

S.L. Pok ${ }^{1}$, F.H. Shabaruddin ${ }^{2}$, J.Y. Teng ${ }^{2}$, M. Dahlui ${ }^{3}$, C.T. $\mathrm{Ng}^{1}$

S. Sockalingam ${ }^{1}$, M.S. Said ${ }^{4}$, A. Rosman ${ }^{5}$, I.S. Lau ${ }^{5}$, L. Mohd Isa ${ }^{6}$,

H. Hussain ${ }^{6}$, S. Mahadeva ${ }^{2} .{ }^{1}$ Dept. of Rheumatology; ${ }^{2}$ Dept. of Medicine;

${ }^{3}$ Dept. of Social \& Preventive Medicine, University of Malaya; ${ }^{4}$ Dept. of

Rheumatology, National University of Malaysia, Kuala Lumpur; ${ }^{5}$ Dept. of

Rheumatology, Selayang Hospital, Selangor; ${ }^{6}$ Dept. of Rheumatology, Putrajaya Hospital, Kuala Lumpur, Malaysia

Background: NSAIDs are frequently used in patients with rheumatoid arthritis (RA) and osteoarthritis (OA). NSAID-induced UGI adverse events are well described in the Western population but data is lacking in Asian patients.

Objectives: To describe the prevalence and direct healthcare costs of NSAIDinduced UGI adverse events in a large cohort of RA and OA patients in Malaysia. Methods: A retrospective cohort study of RA and/or OA patients who received long-term NSAIDs (minimum 4 weeks prescription of any NSAID) between 2010 and 2013 was conducted in 4 large tertiary care centres with rheumatology units in Malaysia. Electronic clinical records and pharmacy prescriptions were reviewed. Resource use data was collected in patients who developed UGI adverse events within the 24 months follow up period. Unit costs were estimated by combining top down (general overheads for hospital services) and bottom up (activity-based costing for clinic visits, hospitalisation, diagnostic investigations, medications) approaches.

Results: 634 patients were included in the final analysis with mean age $53.4 \pm 12.5$ years, $90 \%$ female, diagnosis of RA in $60 \%$, OA in $10 \%$ and both RA and OA in $30 \%$. $45 \%$ and $8 \%$ of patients were on concomitant prednisone and aspirin respectively. $89 \%$ of patients had no previous upper GI disease. $59 \%$ and $41 \%$ of patients were grouped under non-selective and COX-2 inhibitor respectively. $84(13.2 \%)$ patients developed UGl adverse events (Figure 1), consisting of $78(12.3 \%)$ patients with dyspepsia, $5(0.79 \%)$ with peptic ulcer disease (PUD) and $1(0.16 \%)$ with upper Gl bleeding (UGIB). The total direct cost was RM37,352 (USD 11,419) with a mean cost of RM447 \pm 535 (USD 137 \pm 163 ) per patient (Table 1). The largest cost components were pharmacotherapy (34\%), oesophagoduodenoscopies (OGD) (23\%) and outpatient visits (18\%). The mean cost of dyspepsia was RM409 \pm 513 (USD 125 \pm 157 ) per patient. The mean cost of PUD and UGIB was approximately double (RM806 \pm 579 ) (USD 246 \pm 177 ) and quadruple (RM1,602) (USD 490) of dyspepsia respectively.

\begin{tabular}{lcccc}
\hline Healthcare resource & \multicolumn{4}{c}{ Mean cost per patient in RM (USD) } \\
\cline { 2 - 5 } & $\begin{array}{c}\text { Dyspepsia } \\
(\mathrm{n}=78)\end{array}$ & $\begin{array}{c}\text { PUD } \\
(\mathrm{n}=5)\end{array}$ & $\begin{array}{c}\text { UGIB } \\
(\mathrm{n}=1)\end{array}$ & $\begin{array}{c}\text { All patients with UGI } \\
\text { adverse events }(\mathrm{n}=84)\end{array}$ \\
\hline Outpatient visits & $77(23)$ & $146(45)$ & $146(45)$ & $82(25)$ \\
Emergency Dept visits & $28(9)$ & 0 & $146(45)$ & $28(9)$ \\
Inpatient stay & $46(14)$ & $136(41)$ & $679(207)$ & $59(18)$ \\
OGD & $85(26)$ & $253(77)$ & $211(65)$ & $103(32)$ \\
Blood tests & $4(1)$ & $32(10)$ & $57(17)$ & $6(2)$ \\
Radiology & $8(3)$ & 0 & 0 & $8(3)$ \\
Blood transfusion & $4(1)$ & $56(17)$ & $280(86)$ & $10(3)$ \\
Pharmacotherapy & $150(46)$ & $183(56)$ & $84(26)$ & $151(46)$ \\
Mean cost per patient in RM $\pm S D$ & $409 \pm 513$ & $806 \pm 579$ & $1602(490)$ & $447 \pm 535$ \\
$\quad$ (USD) & $(125 \pm 157)$ & $(246 \pm 177)$ & & $(137 \pm 163)$ \\
\hline
\end{tabular}

Conclusions: The low prevalence of UGI adverse events in Malaysian rheuma- 